\title{
Nível de dependência e qualidade de vida da população idosa
}

\author{
Level of dependency and quality of life of elderly \\ Nivel de dependencia y calidad de vida de los ancianos
}

\author{
Alexandrina de Jesus Serra Lobo', Luísa Santos", Sónia Gomes"II \\ 'Escola Superior de Enfermagem Doutor José Timóteo Montalvão Machado. Chaves, Portugal. \\ " Agrupamento de Centros de Saúde Cávado II - Gerês/Cabreira, \\ Unidade de Cuidados de Saúde Personalizados Viver Mais. Amares, Portugal. \\ III Ministério da Saúde, Hospital de Braga. Braga, Portugal.
}

Submissão: 05-01-2013 Aprovação: 24-09-2014

\section{RESUMO}

A qualidade de vida $(Q V)$ nos idosos é determinada em grande parte pelo seu estado funcional e condições de saúde. Com o objectivo de avaliar o nível de QV, os factores que a influenciam e identificar o grau de dependência dos idosos foi realizado um estudo observacional transversal do tipo exploratório-descritivo, englobando 93 idosos. Na recolha de dados utilizouse o índice de Barthel e MOS-SF 36. Na identificação dos níveis de dependência os resultados indicam-nos que $40,0 \%$ são independentes e 18,0\% são dependentes mínimos, sendo 12,0\% dependentes totais. No que diz respeito à QV $88,0 \%$ dos sujeitos refere uma pontuação inferior a 50,0\%, em média reportam uma QV de $39 \pm 10,0 \%$. Verificou-se que existe uma correlação positiva entre o grau de dependência e o índice de QV, sobretudo na componente física. Assim, importa promover um envelhecimento saudável procurando-se privilegiar a preservação da autonomia e capacidade funcional dos idosos.

Descritores: Idosos; Dependência; Qualidade de Vida.

The quality of life $(\mathrm{QoL})$ in older adults is largely determined by their functional status and health conditions. With the purpose of investigate the QoL and the factors affecting it, and identify the degree of dependency of the elderly was carried out an observational cross-sectional exploratory and descriptive, involving 93 elderly. In collecting data we used the Barthel Index and MOS SF-36. In the identification of levels of dependency results indicate us that $40.0 \%$ are independent and $18.0 \%$ are dependents, minimum being $12.0 \%$ total-dependent. The results show us that, $88.0 \%$ of the subjects reported a score below $50.0 \%$ on average reported a QoL of $39 \pm 10.0 \%$. Checking that are a positive correlation between the degree of dependence and the index of QoL, especially in the physical component. It is therefore important to promote healthy aging in an attempt to favor the preservation of autonomy and functional capacity of the elderly.

Key words: Elders; Dependency; Quality of Life.

\section{RESUMEN}

La calidad de vida (CV) en los adultos mayores es en gran parte determinado por su estado funcional y las condiciones de salud. Con el fin de evaluar el nivel de CV y los factores que influyen en ella y determinar el grado de dependencia de los ancianos se llevó a cabo un estudio observacional transversal, exploratorio y descriptivo, que involucró a 93 personas mayores. En la recopilación de datos se utilizó el Índice de Barthel y el MOS SF-36. En la identificación de los nivele de dependiencia los resultados nos indican que $40,0 \%$ son independientes, $18,0 \%$ são dependentes mínimos y $12,0 \%$ dependientes totales. En lo que respeicta a la CV, 88,0\% de los sujetos reportaron una puntuación inferior a 50,0\% en promedio reportó una CV de $39 \pm 10,0 \%$. Tomando nota de que existe una correlación positiva entre el grado de dependencia y el índice de calidad de vida, especialmente en el componente físico. Por tanto, es importante promover un envejecimiento saludable, en un intento de favorecer la preservación de la autonomía y la capacidad funcional de los ancianos.

Palabras clave: Ancianos; Dependencia; Calidad de Vida.

\section{AUTOR CORRESPONDENTE Alexandrina de Jesus Serra Lobo E-mail: damiaolobo@gmail.com}




\section{INTRODUÇÃO}

O envelhecimento é cada vez mais uma realidade demográfica nas sociedades atuais. Na última década assistiu-se a um aumento inédito da população idosa, representando actualmente cerca de $19,0 \%$ da população total, ao qual corresponde um índice de envelhecimento de $129,0 \%{ }^{(1)}$. Espera-se que entre 2010 e 2050 Portugal continue a envelhecer, prevendo-se que a percentagem de idosos venha a duplicar passando para $38,9 \%{ }^{(2)}$. Estima-se que seja o quarto país da União Europeia com maior percentagem de idosos, apenas ultrapassado pela Espanha $(35,6 \%)$, Itália $(35,3 \%)$ e Grécia $(32,5 \%)^{(1)}$.

O envelhecimento constitui um processo biológico inevitável marcado por uma perda progressiva de funções sensoriais e motoras, aumentando consequentemente a vulnerabilidade às doenças. As quais podem afetar a funcionalidade, a mobilidade e a independência, impossibilitando-o de um envelhecimento saudável e autónomo. Segundo Sequeira $(2010)^{(3)} \mathrm{o}$ conceito de dependência relaciona-se com a incapacidade da pessoa para a satisfação das suas necessidades humanas básicas, necessitando da ajuda de terceiros para sobreviver. Veríssimo (2004) $)^{(4)}$ acrescenta que esta "é uma função que varia ao longo da vida implicando reajustamentos criados pelo próprio indivíduo", sendo esta avaliada em função da capacidade de execução das atividades básicas de vida diária.

A maior longevidade do ser humano só faz sentido se corresponder a uma boa qualidade de vida (QV), sendo este um dos principais desafios da sociedade actual. Embora exista alguma confusão entre o estado de saúde física e QV, entende-se que esta se refere a uma consequência directa do próprio estado de saúde. Paralelamente o conceito de saúde alargado à noção de bem-estar sem limites cronológicos, ultrapassa a visão tradicional curativa, preconizando pois, uma abordagem de promoção da saúde, de prevenção da doença e consequentemente um aumento da esperança de vida com um bem viver ${ }^{(4)}$. Neste sentido a QV tem vindo a assumir uma importância crescente, quer no âmbito económico-social, quer no domínio da saúde em geral, tornando-se tanto mais importante quanto mais se desenvolvem as doenças crónicas incapacitantes ${ }^{(5)}$.

A QV é um conceito de amplo alcance afetado de um modo complexo não só pela saúde física, como também pelo estado psicológico, nível de independência, relações sociais e factores ambientais ${ }^{(6)}$. A Organização Mundial de Saúde ${ }^{(6)}$ que conta com um grupo especializado no que diz respeito à $\mathrm{QV}$ definiu o conceito (de acordo com a sua multidimensionalidade) como a perceção do indivíduo sobre a sua posição na vida, no contexto da sua cultura e sistema de valores, em relação com os seus objetivos pessoais, expectativas, padrões e preocupações.

A avaliação da QV dos idosos implica a adopção de múltiplos critérios de natureza biológica, psicológica e sócio estrutural, pois vários elementos são apontados como determinantes ou indicadores de bem-estar na velhice: longevidade, saúde biológica, saúde mental, satisfação, controle cognitivo, competência social, produtividade, actividade, eficácia cognitiva, status social, renda, continuidade de papéis familiares, ocupacionais e continuidade de relações informais com ami$\operatorname{gos}^{(7)}$. Nesta faixa etária, as dependências podem resultar quer de alterações biológicas, quer de mudanças operadas a nível das exigências sociais, sendo que estas últimas parecem determinar as primeiras ${ }^{(8)}$.

A diminuição da capacidade física contribui para o aparecimento da maior parte das doenças crónicas, criando um ciclo vicioso entre as doenças crónicas, a incapacidade funcional e o desempenho físico ${ }^{(9)}$. A este respeito, a maioria dos investigadores que se dedicam às questões relacionadas com o envelhecimento e acima de tudo, com um envelhecimento bem sucedido, referem que, sempre que seja possível, as pessoas idosas devem permanecer no seu próprio ambiente, devendo existir por parte da sociedade, políticas que lhes proporcionem apoio e acompanhamento. Neste âmbito, ao proporcionar-se mais apoio às famílias, aumenta-se a possibilidade destas cuidarem dos seus idosos e de se considerarem intervenientes imprescindíveis num sistema de cuidados partiIhados, auxiliados por profissionais treinados para o efeito ${ }^{(10)}$. Esta será uma resposta que continuará a expandir-se e apresentar-se como a solução ideal para muitos idosos e famílias.

Face ao exposto, entendemos ser pertinente avaliar o nível de QV relacionada com a saúde e os factores que a influenciam, bem como o grau de dependência dos idosos que residem no domicílio. Desta forma procurar-se-á contribuir com indicadores de resultados úteis para a avaliação e intervenção nas necessidades de saúde desta população.

\section{METODOLOGIA}

Foi realizado um estudo observacional transversal do tipo exploratório-descritivo, de natureza quantitativa. Para a identificação da população, idosos das freguesias de Paredes Secas e Caires do Conselho de Amares ( $N=188$ sujeitos), recorreu-se aos dados do Programa Apoio 65 - Idoso em segurança, da polícia de Amares. Foi constituída uma amostra aleatória de 93 idosos, que cumpriam os critérios de inclusão definidos: i) ter 65 anos ou mais; ii) ter capacidade mental para avaliarem e responderem às questões solicitadas, determinada por um score superior ou igual a 24 pontos no Mini Mental State Examination; iii) não ter vínculo a nenhuma instituição de cuidados ou centro social que sirva esse fim; iv) ter residência na área geográfica definida em habitação tipo familiar.

O estudo foi aprovados pela Comissão de Ética para a Saúde (CES) da Administração Regional de Saúde do Norte (ARSN) parecer n. ${ }^{\circ} 88 / 2011^{(11)}$. Neste estudo foram tidos em consideração os princípios éticos da Declaração de Helsínquia, nomeadamente: a não exploração dos participantes, igualdade e justiça no tratamento, a justa seleção da população em estudo, a beneficência, a não-maleficência, a autonomia das pessoas respeitando os seus valores e uma razão risco benefício favorável.

A recolha de dados foi realizada sob a forma de inquérito, aplicado pelos próprios investigadores, nos meses de setembro e novembro de 2011. Foi considerada: i) a caracterização sócio-económica, demográfica e cultural (idade, sexo, estado civil, habilitações literárias; actividade profissional desenvolvida, rendimentos mensais); ii) o índice de dependência operacionalizado pela escala de Barthel(12); iii) e a avaliação da percepção da QV relacionada com a saúde, pela aplicação do MOS SF-36 ${ }^{(13)}$. 


\section{Índice de dependência}

A escala de Barthel ou Índice de Barthel é um instrumento que avalia (in)dependência funcional e a morbilidade em doentes com patologia crónica, indicando se estes necessitam de cuidados ou não. Pretende ainda avaliar se o doente é capaz de desempenhar determinadas tarefas independentemente. A escala é constituída por 10 itens: alimentação, banho, asseio pessoal, vestir-se, controle vesical e intestinal, movimentação, transferência cadeira/cama, mobilidade e subir escadas, o seu total pode variar de 0 a 100 , sendo que inferior a 20 indica dependente total; 20-35 dependente elevado; 40-55 dependente moderada; 60 - 99 dependente mínimo e 100 independente ${ }^{(12)}$. Esta escala foi aplicada em Portugal por Araújo (2007)(14) numa amostra de idosos não institucionalizados, apresentando boas propriedades psicométricas de validade e confiabilidade.

\section{Perceção da qualidade de vida}

O Medical Outcomes Study-Short Form (MOS-SF-36) é um questionário que possibilita avaliar a percepção individual do estado geral da saúde e de QV dos sujeitos, criado por Jonh Ware e Cathy Sherbourne (1992) ${ }^{(15)}$; validado em Portugal por Ferreira (2000) ${ }^{(13)}$, revelando boas propriedades psicométricas (alfa de Cronbach $=0,86$ ).

É constituído por 36 itens repartidos por oito dimensões de estado de saúde, assinalando tanto os estados positivos como os estados negativos de saúde. MOS SF-36 é uma medida genérica do estado de saúde desenhada para ser auto-administrada, ou administrada por entrevista, ou por telefone, sendo actualmente uma das medidas genéricas do estado de saúde mais conhecidas ${ }^{(14)}$. Contempla escalas de itens múltiplos para medir as já referidas oito dimensões de saúde, agrupando-se em duas componentes: a componente física, que compreende a função física (FF), o desempenho físico (DF), a dor corporal (DC) e a saúde em geral (SG) e a componente mental, que engloba o desempenho emocional (DE), a vitalidade (VT), a função social (FS) e a saúde mental (SM). Abarca ainda a escala de transição em saúde, que não sendo considerado um conceito de saúde, pretende medir a mudança em geral na saúde. Para cada uma das dimensões a pontuação é expressa numa escala de 0 (pior estado de saúde possível) a 100 (melhor estado de saúde possível), apresentando um vasto conjunto de resultados que evidenciam a elevada fiabilidade, validade e poder de resposta deste instrumento de medição ${ }^{(13)}$.

\section{TRATAMENTO DE DADOS}

Para o tratamento de dado utilizou-se o Statistical Package for the Social Sciences, versão Windows (SPSS 17.0), empregando estatística descritiva e inferencial. Na descrição e caracterização da amostra utilizaram-se as medidas de tendência central (média) e de dispersão (amplitude e desvio padrão). Utilizou-se o T-teste para comparar os géneros, o One-Way Anova para analisar as diferenças de médias entre os grupos etários, a correlação de Pearson para verificar a existência de associação entre diferentes variáveis. Valores de $p<0,05$ foram considerados estatisticamente significativos.

\section{RESULTADOS}

Na presente amostra ( $n=93 ; 67,0 \%$ são mulheres): os sujeitos apresentam em média 75,6 $6 \pm 7$ anos, com um valor mínimo de 65 e o máximo de 98 anos, sendo que, $46,0 \%$ pertencem ao grupo etário entre os 71 a 80 anos; $56,0 \%$ são casados e $32,0 \%$ são viúvos; a maioria $(54,0 \%)$ apresenta o ensino primário e $44 \%$ são analfabetos; $93,0 \%$ estão aposentados e $86,0 \%$ desempenharam a sua actividade profissional na vida activa no sector primário.

$\mathrm{Na}$ análise dos cuidadores existentes observou-se que: os principais cuidadores informais são os filhos $(14,0 \%)$ e o conjugue $(8,0 \%)$; no que se refere aos cuidadores formais, apurou-se que em 18,0\% dos casos referem-se aos profissionais de enfermagem; existindo 13 casos em famílias de acolhimentos.

$\mathrm{Na}$ análise do nível de QV observou-se um valor mínimo de 15 e um máximo de 69 pontos, correspondendo a uma média de $39 \pm 10$ pontos. Na Figura 1 apresentam-se os resultados obtidos nas diferentes dimensões da QV por género, podendo-se verificar que de um modo geral, o sexo masculino apresenta melhores resultados, sendo essa diferença estatisticaměnte significativa para as componentes FF e DF.

Figura 1 - Resultados das diferentes dimensões da QV por género

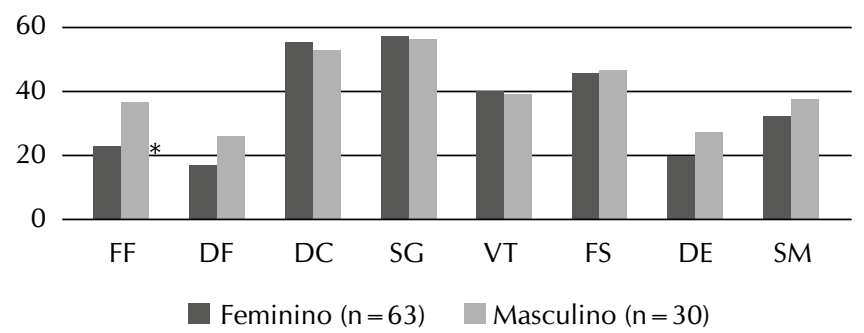

* Diferença estatisticamente significativa $p \leq 0,05$

Quando analisamos esta distribuição por grupos etários, constatou-se que com o aumento da idade os sujeitos tendem a referir pior QV na FF, DF e DE, sendo que os sujeitos com menos de 70 anos apresentam resultados significativamente melhores e em oposição os sujeitos com mais de 91 anos apresentam resultados muito baixos nestas componentes, as quais se acrescenta a DC.

Figura 2 - Resultados das diferentes dimensões da QV por grupos etários

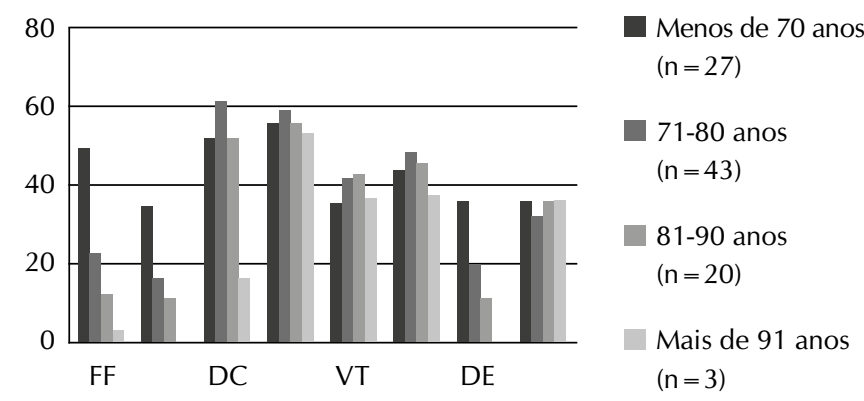

*\# Diferença estatisticamente significativa $p \leq 0,05$ 
Quanto à estatística inferencial, numa primeira análise, cruzamos a pontuação global do SF-36 com as variáveis sociodemográficas que possam influenciar a perceção de QV dos utentes, recorrendo aos testes paramétricos (tabela 1). Quanto ao estado civil (ANOVA: $p=0,007$ ), os casados apresentam melhor QV, observando-se diferenças significativas entre estes e os que apresentam estado civil de solteiros (pos-hoc: $p=0,000$ ) e viúvos (pos-hoc: $p=0,009$ ).

Tabela 1 - Análise inferencial da qualidade de vida global com as variáveis sociodemográficas, Amares, 2011

\begin{tabular}{|c|c|c|c|c|c|}
\hline Variáveis & $\mathbf{n}$ & Media & Teste & $\begin{array}{l}\text { Graus de } \\
\text { liberdade }\end{array}$ & Probabilidade \\
\hline \multicolumn{6}{|l|}{ Qualidade de vida global X } \\
\hline Solteiro & 9 & $31,9^{\mathrm{a}}$ & Anova: & 92 & 0,007 \\
\hline Casado & 53 & $40,5^{\mathrm{A}}$ & $F=5,29$ & & \\
\hline Viúvo & 31 & $35,1^{\mathrm{a}}$ & & & \\
\hline \multicolumn{6}{|l|}{ Habilitações literárias } \\
\hline Não frequentou a escola & 41 & $33,2^{\mathrm{A}}$ & Anova: & 92 & 0,000 \\
\hline $1^{\circ}$ ciclo ensino básico & 50 & $41,6^{\mathrm{a}}$ & $F=9,97$ & & \\
\hline $2^{\circ}$ ciclo ensino básico & 2 & 40,5 & & & \\
\hline \multicolumn{6}{|l|}{ Com quem vive } \\
\hline Sozinho & 15 & $37,9^{a}$ & & & \\
\hline Cônjuge & 49 & $40,7^{\mathrm{a}}$ & Anova: & 92 & 0,000 \\
\hline Filhos & 16 & $36,9^{a}$ & $F=7,29$ & & \\
\hline Família de acolhimento & 13 & $27,4^{\mathrm{A}}$ & & & \\
\hline \multicolumn{6}{|l|}{ Rendimentos mensais } \\
\hline Menos 200euros & 17 & 37,1 & Anova: & 92 & 0,679 \\
\hline Entre 200 e 450 euros & 65 & 37,6 & $F=0,39$ & & \\
\hline Entre 450 e 1000 euros & 11 & 40,2 & & & \\
\hline
\end{tabular}

$A, B, C$ diferenças estatisticamente significativas $(p \leq 0,05)$

Tabela 2 - Correlações entre as variáveis em estudo, Amares, 2011

\begin{tabular}{lccc}
\hline & Nível dependência & Componente Física & Componente mental \\
\hline Idade & $0,48^{* *}$ & $-0,54^{* *}$ & $-0,28^{* *}$ \\
Nível dependência & 1 & $-0,62^{* *}$ & $-0,34^{*}$ \\
\hline
\end{tabular}

**estatisticamente significativa $p \leq 0,01 ;{ }^{*}$ estatisticamente significativa $p \leq 0,05$

Figura 3 - Resultados das diferentes dimensões da QV por níveis de dependência

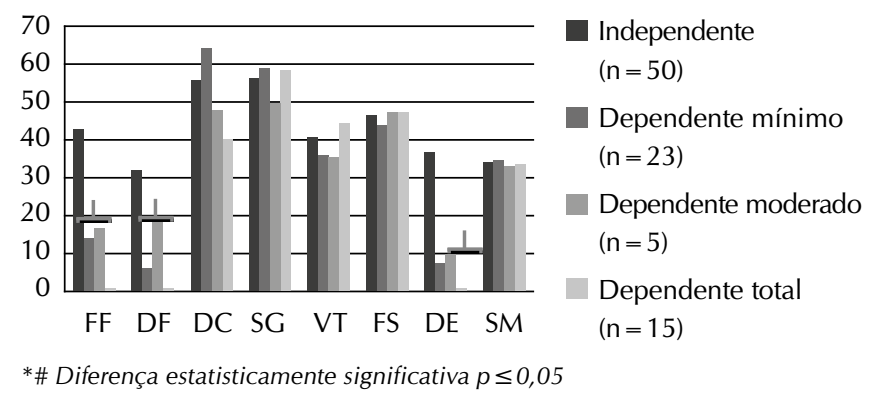

No que se refere às habilitações literárias (ANOVA: $p=0,000$ ), verifica-se que os utentes que não frequentaram a escola têm pior QV, existindo diferenças estatisticamente significativas com os que apresentam o $1^{\circ}$ ciclo de ensino básico (pos-hoc: $p=0,004)$. Por último, em relação à variável com quem vivem, constata-se a existência de diferenças altamente significativas observando-se que os utentes que vivem em famílias de acolhimento percecionam pior QV (ANOVA: $p=0,000$ ). Não se observando diferenças significativas entre os sujeitos de acordo com os rendimentos mensais.

$\mathrm{Na}$ identificação dos níveis de dependência, que corresponde a um dos objetivos principais deste estudo, observou-se que $40 \%$ dos idosos são independentes, $18 \%$ são dependentes mínimos e 12\% dependentes totais. Pela análise da Figura 3, na qual se apresentam os resultados das diferentes dimensões da QV por níveis de dependência constata-se que as componentes mais afectadas pelo nível de dependência são também a FF, DF e DE, sendo esse achado evidente desde a partir da classificação dependente mínimo.

Na Tabela 2 apresentam-se as correlações entre as variáveis em estudo, verificando-se que com o aumento da idade diminui a componente física do SF-36 $(r=-0,54$; $p=0,000)$, aumenta o nível de dependência $(r=0,48 ; p=0,001)$ e por último diminui a componente física do SF-36 ( $r=-0,28 ; p=0,001)$. Observou-se ainda uma forte correlação negativa entre o nível dependência e as componentes físicas e mental do SF-36 $(r=-0,62$; $p=0,001$ e $r=-0,34 ; p=0,040$, respetivamente).

\section{DISCUSSÃO}

Pela análise dos dados sócio económicos e demográficos observou-se que a nossa amostra caracteriza-se por um baixo nível de escolaridade, tendo exercido a sua actividade profissional no sector primário, tal como descrito nos resultados do recenseamento da população portuguesa ${ }^{(1)}$. Estes aspectos "por si" condicionam o acesso à informação, principalmente no que se refere à disponibilidade de cuidados para uma qualquer condição de saúde, afectando a sua perceção de $Q V^{(17-18)}$.

Ainda que se trate de uma questão periférica à nossa investigação, constatou-se que as mulheres são as principais cuidadoras, o que traduz o seu percurso fortemente marcado pelas 
obrigações familiares e domésticas ${ }^{(18)}$. Verificou-se ainda que a maioria dos sujeitos em cuidador informal reporta-se como não tendo QV. A este respeito, Sequeira (2010) ${ }^{(3)}$ reforça a importância dos cuidadores informais na garantia do envelhecimento bem sucedido, visando a manutenção da saúde e do bem-estar dos idosos dependentes.

Genericamente os participantes deste estudo demonstraram uma baixa percepção de QV relacionada com a saúde, evidenciando-se que a mesma é muito afectada pelo nível de dependência, idade avançada e o género. Em concordância com a literatura podemos destacar que o sexo feminino geralmente apresenta uma percepção da QV inferior ao sexo masculino ${ }^{(18-19)}$, sendo que as razões que levam a esta avaliação mais negativa permanecem incertas. Pela análise das pontuações obtidas através da aplicação do SF-36, os resultados indicaram-nos que as componentes mais afectadas são a FF, DF e DE o que não se afasta muito do que é reportado em outros estudos similares ${ }^{(7-8,20)}$. Neste sentido importa clarificar estes conceitos ${ }^{(21)}$, em que a FF diz respeito a avaliação das limitações para execução de actividades físicas ligeiras, como tomar banho ou vestir-se, até às actividades mais vigorosas, passando por actividades intermédias como levantar ou carregar compras da mercearia, subir lanços de escadas, inclinar-se, ajoelhar-se, baixar-se ou mesmo andar uma determinada distância. Paralelamente, a escala do DF e do DE procuram incluir um juízo de valor, auto reportado sobre as implicações das suas limitações, concretamente no tipo e quantidade de trabalho executado, sendo certo que, estes conceitos estão inter-relacionados e actuam sobre a competência funcional do indivíduo.

Mais importante do que a semelhança entre as pontuações interessa a relevância do seu significado. Assim Peterson et al. $(2006)^{(22)}$, verificou-se que nesta faixa etária especialmente sensível às limitações funcionais e à degeneração da saúde, os aspectos da condição física pesam consideravelmente na noção de QV. Paralelamente, os estudos indicam que os idosos tendem a ter uma vida isolada, com poucas oportunidades de convívio e socialização, que de uma forma ou outra os pode afectar psicologicamente, podendo-se reflectir no DE, na negligência para o autocuidado e na perda de autonomia ${ }^{(22)}$.

De acordo com alguns trabalhos, tal como no presente estudo verificou-se uma relação negativa entre a idade e a percepção subjectiva de saúde, indicando que os défices na função física e cognitiva, associados ao avanço da idade, estão entre os preditores primários da diminuição da QV nos idosos $^{(23)}$. Também num estudo realizado pela University of
California, Keeler (2010)(24) demonstrou-se que o estado funcional tem um forte impacto na qualidade e expectativa de vida dos idosos referindo que: um idoso de 75 anos independente tem uma expectativa de vida 5 anos maior do que o idoso da mesma idade com dependência para AVD; e a expectativa de vida de um idoso dependente de 75 anos é similar a de um idoso de 85 anos e independente.

A este aspeto acrescenta-se ainda o facto do nível de dependência se associar negativamente com a QV. Dentro desta linha de pensamento, com o avanço da idade a QV diminuiu mesmo em adultos saudáveis, podendo resultar num aumento da incidência de incapacidade para o uso de serviços e cuidados médicos, a perda de independência e a redução da $Q V^{(25)}$.

Apesar dos resultados deste estudo serem consonantes com os já existentes, apontamos como principal limitação, o reduzido tamanho da amostra. No entanto, procurou-se selecionar uma amostra aleatória que se pode considerar representativa para a análise do nível de dependência e QV desta população, já que para esta análise há que considerar múltiplos factores que se inter-relacionam, acrescentando-se ainda o facto deste grupo etário ser muito heterogéneo, dificultando a generalização dos resultados. Contudo com este estudo, ampliou-se o conhecimento que se objectiva necessário para o desenvolvimento de acções de prevenção e/ou minimização da morbimortalidade, no sentido de melhorar a QV da população em geral e dos idosos em particular. A existência de cuidadores informais e o nível de dependência dos sujeitos são os principais determinantes identificados. Esta problemática exige uma mudança política e social, onde se privilegie a atenção e acompanhamento dos cuidadores informais que garanta a promoção de ambientes facilitadores de autonomia e independência. Na vertente preventiva releva-se a importância de fomentar a participação dos idosos nas actividades de vida diária e na manutenção de estilos de vida mais activos, como sendo essenciais para a diminuição da dependência e aumento da sua expectativa face à QV.

\section{CONSIDERAÇÕES FINAIS}

O significado atribuído à QV tende a transformar-se ao longo da trajectória pessoal do indivíduo, não por influência da idade em "si mesma", mas essencialmente devido a acontecimentos, mudanças e experiencias que acabam por se associar ao avanço da idade, onde se destaca o contexto familiar e as alterações de saúde.

\section{REFERÊNCIAS}

1. Instituto Nacional de Estatística (PT). Recenseamento Geral da População [Internet]. Lisboa: Instituto Nacional de Estatística; 2011 [acesso em 03 de dezembro de 2011]. Disponível em: http://censos.ine.pt/xportal/ xmain? $x$ pid $=$ CENSOS\&xpgid $=$ ine_censos_publicacoes

2. Europedirect [Internet]. [place unknown: publisher unknown]; [updated 2014 November 18; cited 2011 December 03]. Available from: http://www.europedirect.uac.pt
3. Sequeira C. Cuidar de idosos com dependência física e mental. Lisboa-Porto: Lidel- Edições técnicas limitadas; 2010.

4. Veríssimo CMF. Importância dos conceitos para a produção multidisciplinar de cuidados. Nursing. 2004;15(187):20-4.

5. Ioshimoto T. Dependência funcional, mortalidade e qualidade de vida nos Idosos [editorial]. Rev Neurociênc [Internet]. 2010 [acesso em 03 de dezembro de 2011];18(4):423-4. 
Disponível em: http://www.revistaneurociencias.com.br/ edicoes/2010/RN1804/editorial\%20thais.pdf

6. The world health organization quality of life assessment (WHOQOL): position paper from the world health organization. SocSci Med [Internet]. 1995 [cited 2011 December 03];41(10):1403-9. Available from: http://www.ncbi. nlm.nih.gov/pubmed/8560308

7. Pereira RJ, Cotta RMMC, Franceschini SCC, Ribeiro RCL, Sampaio RF, Priore SE, et al. Contribuição dos domínios físico, social, psicológico e ambiental para a qualidade de vida global de idosos. Rev Psiquiatr Rio Gd Sul [Internet]. 2006 [acesso em 03 de dezembro de 2011];28(1):27-38. Disponível em: http://www.scielo.br/pdf/rprs/v28n1/v28n1a05.pdf

8. Torres GV, Reis LA, Reis LA, Fernandes MH. Qualidade de vida e fatores associados em idosos dependentes em uma cidade do interior do Nordeste. J Bras Psiquiatr. 2009;58(1):39-44

9. Ferrucci L. The Journals of Gerontology. Series A, Biological Sciences and Medical Sciences. J Gerontology. 2007;62A(9):989-96.

10. Martins R. Envelhecimento e políticas sociais [Internet]. [lugar desconhecido]: Educação, ciência e tecnologia; 2006 [acesso em 13 de setembro de 2011. Disponível em: http://www.ipv.pt/millenium/Millenium32/10.pdf

11. Comissão de Ética para a Saúde (BR). Parecer $n^{\circ} .88 / 2011$ da CES [Internet]. [acesso em 13 de setembro de 2011]. Disponível em: http://portal.arsnorte.min-saude.pt/portal/page/portal/ARSNorte/Comiss $\%$ C $3 \%$ A3o $\% 20 \mathrm{de} \% 20$ $\%$ C3\%89tica/Ficheiros/Parecer CES 88 2011.pdf

12. Mahoney FI, Barthel DW. Functional evaluation: the Barthel Index. Md State Med J [Internet]. 1965 [cited 2011 September 13];14:61-65. Available from: http://www. ncbi.nlm.nih.gov/pubmed/14258950

13. Ferreira PL. Criação da versão portuguesa do MOS SF-36: parte I - Adaptação cultural e linguística. ActMedPort [Internet]. 2000 [acesso 03 dezembro de 2011];13(1-2):55-63. Disponível em: file:///C:/Users/Cliente/Downloads/17602414-1-PB.pdf

14. Araújo F, Ribeiro JLP, Oliveira A, Pinto C. Validação do índice de Barthel numa amostra de idosos não institucionalizados. Rev Port Saúde Pública [Internet]. 2007 [acesso 03 de dezembro de 2011];25(2):59-66. Disponível em: http://repositorio-aberto.up.pt/bitstream/10216/15740/2/52442.pdf

15. Ware JE Jr, Sherbourne CD. The MOS 36-item short- form health survey (SF-36). I. Conceptual framework and item selection. Med Care [Internet]. 1992 [cited 2011 September 13]. Available from: http://www.ncbi.nlm.nih.gov/ pubmed/1593914

16. Inouye K, Pedrazzani E. Nível de instrução, status socioeconómico e avaliação de algumas dimensões da qualidade de vida de octagenários. Rev Latinoam Enferm. 2007;15(nº. esp.):742-7.
17. Agostinho F, Vilela TR, Carvalho GA. Determinantes da qualidade de vida em idosos diabéticos. Rev Digital [Internet]. 2008 [acesso 03 dezembro de 2011];13(124). Disponível em: C:IUsers\ClientelDownloads\Determinantes da qualidade de vida em idosos diabéticos (2).mht

18. Millán-Calenti JC, Tubío J, Pita-Fernández S, González-Abraldes I, Lorenzo T, Fernández-Arruty T, et al. Prevalence of functional disability in activities of daily living (ADL), instrumental activities of daily living (IADL) and associated factors, as predictors of morbidity and mortality. Arch GerontolGeriatr [Internet]. 2010 [cited 2011 September 13];50(3):306-10. Available from: http://www. ncbi.nlm.nih.gov/pubmed/19520442

19. Aires M, Paskulin LMG, Morais EP. Capacidade funcional de idosos mais velhos: estudo comparativo em três regiões do Rio Grande do Sul]. Rev Latinoam Enferm [Internet]. 2010 [acesso 03 dezembro de 2011];18(1):[07 telas] Disponível em http://www.scielo.br/pdf/rlae/v18n1/pt_03.pdf

20. Turner JA, Fulton-Kehoe D, Franklin G, Wickizer TM, Wu R. Comparison of the Roland-Morris Disability Questionnaire and generic health status measures: a populationbased study of workers' compensation back injury claimants. Spine (Phila Pa 1976) [Internet]. 2003 [cited 2011 September 13];28(10):1061-7. Available from: http:// www.ncbi.nlm.nih.gov/pubmed/12768149

21. Salgueiro HDAG. Perceção do estado de saúde e de qualidade de vida da família que coabita e cuida de um idoso dependente. Rev Investigação Enfermagem [Internet]. 2008 [acesso 03 dezembro de 2011];(17):51-63. Disponível em: http://sinaisvitais.pt/index.php/revistas/00revista-investigacao-em-enfermagem/31-2008-a-2011/350-revista-de-investiga-no-17-parte-2 ? showall $=\&$ start $=4$

22. Peterson JJ, Lowe JB, Peterson NA, Janz KF. The relationship between active living and health-related quality of life: income as a moderator. Health Educ Res [Internet]. 2006 [cited 2011 September 13];21(1):146-56. Available from: http://www.ncbi.nlm.nih.gov/pubmed/16087691

23. Cassidy K, Kotynia-English R, Acres J, Flicker L, Lautenschlager NT, Almeida OP. Association between lifestyle factors and mental health measures among community-dwelling older women. Austr N Z J Psychiatry [Internet]. 2004 [cited 2011 September 13];38(1112):940-7. Available from: http://www.ncbi.nlm.nih.gov/ pubmed/15555029

24. Keeler E, Guralnik JM, Tian H, Wallace RB, Reuben DB. The impact of functional status on life expectancy in older persons. J Gerontol A BiolSci Med Sci [Internet]. 2010 [cited 2011 September 13];65(7):727-33. Available from: http://www.ncbi.nlm.nih.gov/pubmed/20363833

25. Jackson AS. The evolution and validity of Health-Related Fitness. Quest [Internet]. 2006 [cited 2011 September 13];58(1):160-75. Available from: http://www.tandfonline.com/doi/abs/10.1080/00336297.2006.10491877\#. VEZolPIdWaY 